\title{
Glass physics: from fundamentals to applications
}

\author{
Published online: 22 September 2011 - (c) EDP Sciences / Società Italiana di Fisica / Springer-Verlag 2011
}

\begin{abstract}
A new focus on glass physics has arisen with the emergence, on the one hand, of soft glassy materials in technologically significant and biological contexts, and, on the other hand, of metallic glasses that present intriguing opportunities for the efficient manufacture of valuable high strength components. The slow dynamics that develops in liquids cooled toward the glass transition temperature and the nature of the glass transition that follows remain at the core of the glass puzzle, and endure as grand theoretical challenges in understanding condensed matter. However, a focus on the applications of these new materials has resulted in a flowering of investigations on the origin of aging, dynamical heterogeneity and slow relaxation with a focus on relating glass structure to the prediction of important properties such as fragility, glass formability, rheology and elastic and plastic responses to mechanical stress. Simultaneously the scope of research has expanded in new directions owing to advances in research tools ranging from more powerful computational capabilities to improved scattering facilities and advanced imaging techniques. Sustained engagement between physical scientists and the materials science communities working to unlock the technological capabilities of specific glasses has generated a renewed interest in previously underappreciated gaps in our understanding, expanding the productive interface between fundamental and applied investigations in glass physics.
\end{abstract}

This special issue in European Physical Journal E brings together a cross-section of research advances, highlighting the diversity of basic and applied interest in glassy behavior in a wide range of systems (colloidal, polymer, chalcogenide, metallic, granular, biomolecular, organic and oxide glasses). Several papers discuss theoretical and computational investigations of model glass formers such as a critique of structure-based microscopic theories of dynamics and a field-theoretic description of dynamical heterogeneity. The papers in this issue showcase examples of cross-fertilization, such as the application of mode-coupling theory, extensively employed in analyzing slow dynamics in glass-forming liquids, to analyzing motion under shear, but also energy-landscape analysis of mechanical deformation, the study of heterogeneity effects in polymer thin films and the jamming behavior in polymer solutions, to name but a few examples.

We hope that this compilation will serve the purpose of furthering the interaction across the boundaries between fundamental and applied research, and among practitioners in different sub-areas, leading to the emergence of new thrust directions, concepts, and exciting developments.

Michael Falk, Takeshi Egami and Srikanth Sastry 